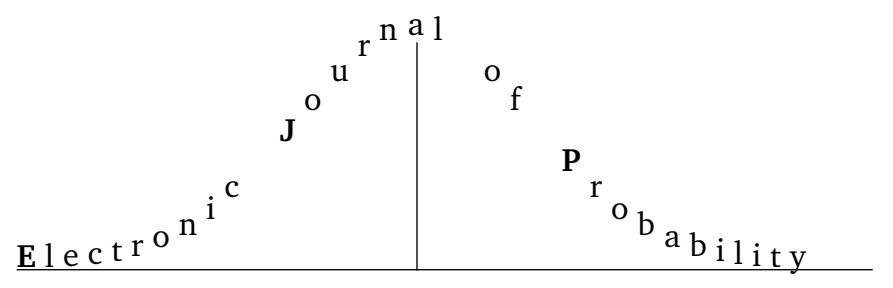

Vol. 15 (2010), Paper no. 22, pages 684-709.

Journal URL

http://www.math.washington.edu/ ejpecp/

\title{
Poisson-type processes governed by fractional and higher-order recursive differential equations
}

\author{
L.Beghin* $\quad$ E.Orsingher ${ }^{\dagger}$
}

\begin{abstract}
We consider some fractional extensions of the recursive differential equation governing the Poisson process, i.e.

$$
\frac{d}{d t} p_{k}(t)=-\lambda\left(p_{k}(t)-p_{k-1}(t)\right), \quad k \geq 0, t>0
$$

by introducing fractional time-derivatives of order $v, 2 v, \ldots, n v$. We show that the so-called "Generalized Mittag-Leffler functions" $E_{\alpha, \beta}^{k}(x), x \in \mathbb{R}$ (introduced by Prabhakar [24]) arise as solutions of these equations. The corresponding processes are proved to be renewal, with density of the intearrival times (represented by Mittag-Leffler functions) possessing power, instead of exponential, decay, for $t \rightarrow \infty$. On the other hand, near the origin the behavior of the law of the interarrival times drastically changes for the parameter $v$ varying in $(0,1]$.

For integer values of $v$, these models can be viewed as a higher-order Poisson processes, connected with the standard case by simple and explict relationships.
\end{abstract}

Key words: Fractional difference-differential equations; Generalized Mittag-Leffler functions; Fractional Poisson processes; Processes with random time; Renewal function; Cox process.

AMS 2000 Subject Classification: Primary 60K05; 33E12; 26A33.

Submitted to EJP on November 4, 2009, final version accepted April 14, 2010.

\footnotetext{
*Sapienza University of Rome

†Corresponding author. Address: Sapienza University of Rome, p.le A. Moro 5, 00185, Rome, Italy. E-mail: enzo.orsingher@uniroma1.it
} 


\section{Introduction}

Many well-known differential equations have been extended by introducing fractional-order derivatives with respect to time (for instance, the heat and wave equations ([14]-[15] and [21]) as well as the telegraph equation ([20]) and the higher-order heat-type equations ([1])) or with respect to space (for instance, the equations involving the Riesz fractional operator).

Fractional versions of the Poisson processes have been already presented and studied in the literature: in [10] the so-called fractional master equation was considered. A similar model was treated in [13], where the equation governing the probability distribution of the homogeneous Poisson process was modified, by introducing the Riemann-Liouville fractional derivative. The results are given in analytical form, in terms of infinite series or successive derivatives of Mittag-Leffler functions. We recall the definition of the (two-parameter) Mittag-Leffler function:

$$
E_{\alpha, \beta}(x)=\sum_{r=0}^{\infty} \frac{x^{r}}{\Gamma(\alpha r+\beta)}, \quad \alpha, \beta \in \mathbb{C}, \operatorname{Re}(\alpha), \operatorname{Re}(\beta)>0, x \in \mathbb{R},
$$

(see [22], $\breve{g} 1.2$ ).

Another approach was followed by Repin and Saichev [25]: they start by generalizing, in a fractional sense, the distribution of the interarrival times $U_{j}$ between two successive Poisson events. This is expressed, in terms of Mittag-Leffler functions, for $v \in(0,1]$, as follows:

$$
f(t)=\operatorname{Pr}\left\{U_{j} \in d t\right\} / d t=-\frac{d}{d t} E_{v, 1}\left(-t^{v}\right)=\sum_{m=1}^{\infty} \frac{(-1)^{m+1} t^{v m-1}}{\Gamma(v m)}, \quad t>0
$$

and coincides with the solution to the fractional equation

$$
\frac{d^{v} f(t)}{d t^{v}}=-f(t)+\delta(t), \quad t>0
$$

where $\delta(\cdot)$ denotes the Dirac delta function and again the fractional derivative is intended in the Riemann-Liouville sense. For $v=1$ formula (1.2) reduces to the well-known density appearing in the case of a homogeneous Poisson process, $N(t), t>0$, with intensity $\lambda=1$, i.e. $f(t)=e^{-t}$.

The same approach is followed by Mainardi et al. [17]-[18]-[19], where a deep analysis of the related process is performed: it turns out to be a true renewal process, loosing however the Markovian property. Their first step is the study of the following fractional equation (instead of (1.3))

$$
\frac{d^{v} \psi(t)}{d t^{v}}=-\psi(t)
$$

with initial condition $\psi\left(0^{+}\right)=1$ and with fractional derivative defined in the Caputo sense. The solution $\psi(t)=E_{v, 1}\left(-t^{v}\right)$ to $(1.4)$ represents the survival probability of the fractional Poisson process. As a consequence its probability distribution is expressed in terms of derivatives of MittagLeffler functions, while the density of the $k$-th event waiting time is a fractional generalization of the Erlang distribution and coincides with the $k$-fold convolution of $(1.2)$.

The fractional Poisson process (a renewal process with Mittag-Leffler intertime distribution) has proved to be useful in several fields, like the analysis of the transport of charged carriers (in [30]), 
in finance (in [16]) and in optics to describe the light propagation through non-homogeneous media (see [5]).

The analysis carried out by Beghin and Orsingher [2] starts, as in [13], from the generalization of the equation governing the Poisson process, where the time-derivative is substituted by the fractional derivative (in the Caputo sense) of order $v \in(0,1]$ :

$$
\frac{d^{v} p_{k}}{d t^{v}}=-\lambda\left(p_{k}-p_{k-1}\right), \quad k \geq 0
$$

with initial conditions

$$
p_{k}(0)= \begin{cases}1 & k=0 \\ 0 & k \geq 1\end{cases}
$$

and $p_{-1}(t)=0$. The main result is the expression of the solution as the distribution of a composed process represented by the standard, homogeneous Poisson process $N(t), t>0$ with a random time argument $\mathscr{T}_{2 v}(t), t>0$ as follows:

$$
\mathscr{N}_{v}(t)=N\left(\mathscr{T}_{2 v}(t)\right), \quad t>0 .
$$

The process $\mathscr{T}_{2 v}(t), t>0$ (independent of $N$ ) possesses a well-known density, which coincides with the folded solution to a fractional diffusion equation of order $2 v$ (see (2.8) below). In the particular case where $v=1 / 2$ this equation coincides with the heat-equation and the process representing time is the reflected Brownian motion.

These results are reconsidered here, in the next section, from a different point of view, which is based on the use of the Generalized Mittag-Leffler (GML) function. The latter is defined as

$$
E_{\alpha, \beta}^{\gamma}(z)=\sum_{r=0}^{\infty} \frac{(\gamma)_{r} z^{r}}{r ! \Gamma(\alpha r+\beta)}, \quad \alpha, \beta, \gamma \in \mathbb{C}, \operatorname{Re}(\alpha), \operatorname{Re}(\beta), \operatorname{Re}(\gamma)>0
$$

where $(\gamma)_{r}=\gamma(\gamma+1) \ldots(\gamma+r-1)$ (for $r=1,2, \ldots$, and $\gamma \neq 0$ ) is the Pochammer symbol and $(\gamma)_{0}=1$. The GML function has been extensively studied by Saxena et al. (see, for example, [27]-[28]) and applied in connection with some fractional diffusion equations, whose solutions are expressed as infinite sums of (1.6). For some properties of (1.6), see also [29]. We note that formula (1.6) reduces to (1.1) for $\gamma=1$.

By using the function (1.6) it is possible to write down in a more compact form the solution to (1.5), as well as the density of the waiting-time of the $k$-th event of the fractional Poisson process. As a consequence some interesting relationships between the Mittag-Leffler function (1.1) and the GML function (1.6) are obtained here.

Moreover, the use of GML functions allows us to derive an explicit expression for the solution of the more complicated recursive differential equation, where two fractional derivatives appear:

$$
\frac{d^{2 v} p_{k}}{d t^{2 v}}+2 \lambda \frac{d^{v} p_{k}}{d t^{v}}=-\lambda^{2}\left(p_{k}-p_{k-1}\right), \quad k \geq 0,
$$

for $v \in(0,1]$. As we will see in section 3 , also in this case we can define a process $\widehat{N_{v}}(t), t>0$, governed by (1.7), which turns out to be a renewal. The density of the interarrival times are no-longer expressed by standard Mittag-Leffler functions as in the first case, but the use of GML functions is required and the same holds for the waiting-time of the $k$-th event. 
An interesting relationship between the two models analyzed here can be established by observing that the waiting-time of the $k$-th event of the process governed by (1.7) coincides in distribution with the waiting time of the $(2 k)$-th event for the first model. This suggests to interpret $\widehat{N_{v}}$ as a fractional Poisson process of the first type, which jumps upward at even-order events $A_{2 k}$ and the probability of the successive odd-indexed events $A_{2 k+1}$ is added to that of $A_{2 k}$. As a consequence, the distribution of $\widehat{\mathscr{N}_{v}}$ can be expressed, in terms of the processes $N$ and $\mathscr{T}_{2 v}$, as follows:

$$
\operatorname{Pr}\left\{\widehat{\mathscr{N}_{v}}(t)=k\right\}=\operatorname{Pr}\left\{N\left(\mathscr{T}_{2 v}(t)\right)=2 k\right\}+\operatorname{Pr}\left\{N\left(\mathscr{T}_{2 v}(t)\right)=2 k+1\right\}, \quad k \geq 0 .
$$

We also study the probability generating functions of the two models, which are themselves solutions to fractional equations; in particular in the second case an interesting link with the fractional telegraph-type equation is explored.

For $v=1$, equation (1.7) takes the following form

$$
\frac{d^{2} p_{k}}{d t^{2}}+2 \lambda \frac{d p_{k}}{d t}=-\lambda^{2}\left(p_{k}-p_{k-1}\right), \quad k \geq 0
$$

and the related process can be regarded as a standard Poisson process with Gamma-distributed interarrival times (with parameters $\lambda, 2$ ). This is tantamount to attributing the probability of oddorder values $A_{2 k+1}$ of a standard Poisson process to the events labelled by $2 k$. Moreover, it should be stressed that, in this special case, the equation satisfied by the probability generating function $\widehat{G}(u, t), t>0,|u| \leq 1$, i.e.

$$
\frac{\partial^{2} G(u, t)}{\partial t^{2}}+2 \lambda \frac{\partial G(u, t)}{\partial t}=\lambda^{2}(u-1) G(u, t), \quad 0<v \leq 1
$$

coincides with that of the damped oscillations.

All the previous results are further generalized to the case $n>2$ in the concluding remarks: the structure of the process governed by the equation

$$
\frac{d^{n v} p_{k}}{d t^{n v}}+\left(\begin{array}{c}
n \\
1
\end{array}\right) \lambda \frac{d^{(n-1) v} p_{k}}{d t^{(n-1) v}}+\ldots+\left(\begin{array}{c}
n \\
n-1
\end{array}\right) \lambda^{n-1} \frac{d^{v} p_{k}}{d t^{v}}=-\lambda^{n}\left(p_{k}-p_{k-1}\right), \quad k \geq 0,
$$

where $v \in(0,1]$, is exactly the same as before and all the previous considerations can be easily extended.

\section{First-type fractional recursive differential equation}

\subsection{The solution}

We begin by considering the following fractional recursive differential equation

$$
\frac{d^{v} p_{k}}{d t^{v}}=-\lambda\left(p_{k}-p_{k-1}\right), \quad k \geq 0
$$

with $p_{-1}(t)=0$, subject to the initial conditions

$$
p_{k}(0)=\left\{\begin{array}{ll}
1 & k=0 \\
0 & k \geq 1
\end{array} .\right.
$$


We apply in (2.1) the definition of the fractional derivative in the sense of Caputo, that is, for $m \in \mathbb{N}$,

$$
\frac{d^{v}}{d t^{v}} u(t)=\left\{\begin{array}{l}
\frac{1}{\Gamma(m-v)} \int_{0}^{t} \frac{1}{(t-s)^{1+v-m}} \frac{d^{m}}{d s^{m}} u(s) d s, \quad \text { for } m-1<v<m \\
\frac{d^{m}}{d t^{m}} u(t), \quad \text { for } v=m
\end{array} .\right.
$$

We note that, for $v=1$, (2.1) coincides with the equation governing the homogeneous Poisson process with intensity $\lambda>0$.

We will obtain the solution to (2.1)-(2.2) in terms of GML functions (defined in (1.6)) and show that it represents a true probability distribution of a process, which we will denote by $\mathscr{N}_{v}(t), t>0$ : therefore we will write

$$
p_{k}^{v}(t)=\operatorname{Pr}\left\{\mathscr{N}_{v}(t)=k\right\}, \quad k \geq 0, t>0
$$

Theorem 2.1 The solution $p_{k}^{v}(t)$, for $k=0,1, \ldots$ and $t \geq 0$, of the Cauchy problem (2.1)-(2.2) is given by

$$
p_{k}^{v}(t)=\left(\lambda t^{v}\right)^{k} E_{v, v k+1}^{k+1}\left(-\lambda t^{v}\right), \quad k \geq 0, t>0 .
$$

Proof By taking the Laplace transform of equation (2.1) together with the condition (2.2), we obtain

$$
\mathscr{L}\left\{p_{k}^{v}(t) ; s\right\}=\int_{0}^{\infty} e^{-s t} p_{k}^{v}(t) d t=\frac{\lambda^{k} s^{v-1}}{\left(s^{v}+\lambda\right)^{k+1}}
$$

which can be inverted by using formula (2.5) of [24], i.e.

$$
\mathscr{L}\left\{t^{\gamma-1} E_{\beta, \gamma}^{\delta}\left(\omega t^{\beta}\right) ; s\right\}=\frac{s^{\beta \delta-\gamma}}{\left(s^{\beta}-\omega\right)^{\delta}},
$$

(where $\operatorname{Re}(\beta)>0, \operatorname{Re}(\gamma)>0, \operatorname{Re}(\delta)>0$ and $s>|\omega|^{\frac{1}{\operatorname{Re}(\beta)}}$ ) for $\beta=v, \delta=k+1$ and $\gamma=v k+1$. Therefore the inverse of (2.6) coincides with (2.5).

Remark 2.1 For any $v \in(0,1]$, it can be easily seen that result (2.5) coincides with formula (2.10) of [2], which was obtained by a different approach.

Moreover Theorem 2.1 shows that the first model proposed by Mainardi et al. [17] as a fractional version of the Poisson process (called renewal process of Mittag-Leffler type) has a probability distribution coinciding with the solution of equation (2.1) and therefore with (2.5).

We derive now an interesting relationship between the GML function in (2.5) and the Wright function

$$
W_{\alpha, \beta}(x)=\sum_{k=0}^{\infty} \frac{x^{k}}{k ! \Gamma(\alpha k+\beta)}, \quad \alpha>-1, \beta>0, x \in \mathbb{R} .
$$

Let us denote by $v_{2 v}=v_{2 v}(y, t)$ the solution to the Cauchy problem

$$
\left\{\begin{array}{lc}
\frac{\partial^{2 v} v}{\partial t^{2 v}}=\lambda^{2} \frac{\partial^{2} v}{\partial y^{2}}, & t>0, y \in \mathbb{R} \\
v(y, 0)=\delta(y), & \text { for } 0<v<1 \\
v_{t}(y, 0)=0, & \text { for } 1 / 2<v<1
\end{array} .\right.
$$


then it is well-known (see [14] and [15]) that the solution of (2.8) can be written as

$$
v_{2 v}(y, t)=\frac{1}{2 \lambda t^{v}} W_{-v, 1-v}\left(-\frac{|y|}{\lambda t^{v}}\right), \quad t>0, y \in \mathbb{R}
$$

In [2] the following subordinating relation has been proved:

$$
p_{k}^{v}(t)=\int_{0}^{+\infty} e^{-y} \frac{y^{k}}{k !} \bar{v}_{2 v}(y, t) d y=\operatorname{Pr}\left\{N\left(\mathscr{T}_{2 v}(t)\right)=k\right\}, \quad k \geq 0,
$$

where

$$
\bar{v}_{2 v}(y, t)=\left\{\begin{array}{l}
2 v_{2 v}(y, t), \\
0, \quad y<0
\end{array} \quad y>0\right.
$$

is the folded solution of equation (2.8). In (2.10) $\mathscr{T}_{2 v}(t), t>0$ represents a random time (independent from the Poisson process $N$ ) with transition density given in (2.9) and (2.11). This density can be alternatively expressed in terms of the law $g_{v}(\cdot ; y)$ of a a stable random variable $S_{v}(\mu, \beta, \sigma)$ of order $v$, with parameters $\mu=0, \beta=1$ and $\sigma=\left(\frac{y}{\lambda} \cos \frac{\pi v}{2}\right)^{\frac{1}{v}}$, as

$$
\bar{v}_{2 v}(y, t)=\frac{1}{\Gamma(1-v)} \int_{0}^{t}(t-w)^{-v} g_{v}\left(w ; \frac{y}{\lambda}\right) d w
$$

(see [20], formula (3.5), for details). By combining (2.5) and 2.10), we extract the following integral representation of the GML functions, in terms of Wright functions:

$$
E_{v, v k+1}^{k+1}\left(-\lambda t^{v}\right)=\frac{1}{k ! \lambda^{k+1} t^{v(k+1)}} \int_{0}^{+\infty} e^{-y} y^{k} W_{-v, 1-v}\left(-\frac{y}{\lambda t^{v}}\right) d y .
$$

Remark 2.2 Since result (2.13) holds for any $t>0$, we can choose $t=1$, so that we get, by means of a change of variable,

$$
E_{v, v k+1}^{k+1}(-\lambda)=\frac{1}{k !} \int_{0}^{+\infty} e^{-\lambda y} y^{k} W_{-v, 1-v}(-y) d y .
$$

This shows that the GML function $E_{v, v k+1}^{k+1}$ can be interpreted as the Laplace transform of the function $\frac{y^{k}}{k !} W_{-v, 1-v}(-y)$. In particular, for $v=\frac{1}{2}$, since 2.10 reduces to

$$
\operatorname{Pr}\left\{\mathscr{N}_{1 / 2}(t)=k\right\}=\int_{0}^{+\infty} e^{-y} \frac{y^{k}}{k !} \frac{e^{-y^{2} / 4 \lambda^{2} t}}{\sqrt{\pi \lambda^{2} t}} d y=\operatorname{Pr}\left\{N\left(\left|B_{\lambda}(t)\right|\right)=k\right\},
$$

where $B_{\lambda}(t)$ is a Brownian motion with variance $2 \lambda^{2} t$ (independent of $N$ ), we get (for $t=1$ )

$$
E_{\frac{1}{2}, \frac{k}{2}+1}^{k+1}(-\lambda)=\frac{1}{k !} \int_{0}^{+\infty} e^{-\lambda y} y^{k} \frac{e^{-y^{2} / 4}}{\sqrt{\pi}} d y .
$$

The previous relation can be checked directly, by performing the integral in (2.14). 


\subsection{Properties of the corresponding process}

From the previous results we can conclude that the GML function $E_{v, v k+1}^{k+1}\left(-\lambda t^{v}\right), k \geq 0$, suitably normalized by the factor $\left(\lambda t^{v}\right)^{k}$, represents a proper probability distribution and we can indicate it as $\operatorname{Pr}\left\{\mathscr{N}_{v}(t)=k\right\}$.

Moreover by (2.10) we can consider the process $\mathscr{N}_{v}(t), t>0$ as a time-changed Poisson process. It is well-known (see [12]) that, for a homogeneous Poisson process $N$ subject to a random time change (by the random function $\Lambda((0, t])$ ), the following equality in distribution holds:

$$
N(\Lambda((0, t])) \stackrel{d}{=} M(t)
$$

where $M(t), t>0$ is a Cox process directed by $\Lambda$. In our case the random measure $\Lambda((0, t])$ possesses distribution $\bar{v}_{2 v}$ given in (2.9) with (2.11) and we can conclude that $\mathscr{N}_{v}$ is a Cox process. This conclusion will be confirmed by the analysis of the factorial moments.

Moreover, as remarked in [2] and [18], the fractional Poisson process $\mathscr{N}_{v}(t), t>0$ represents a renewal process with interarrival times $U_{j}$ distributed according to the following density, for $j=$ $1,2, \ldots:$

$$
f_{1}^{v}(t)=\operatorname{Pr}\left\{\mathscr{U}_{j} \in d t\right\} / d t=\lambda t^{v-1} E_{v, v}\left(-\lambda t^{v}\right)
$$

with Laplace transform

$$
\mathscr{L}\left\{f_{1}^{v}(t) ; s\right\}=\frac{\lambda}{s^{v}+\lambda} .
$$

Therefore the density of the waiting time of the $k$-th event, $T_{k}=\sum_{j=1}^{k} U_{j}$, possesses the Laplace transform

$$
\mathscr{L}\left\{f_{k}^{v}(t) ; s\right\}=\frac{\lambda^{k}}{\left(s^{v}+\lambda\right)^{k}} .
$$

Its inverse can be obtained by applying again (2.7) for $\beta=v, \gamma=v k$ and $\omega=-\lambda$ and can be expressed, as for the probability distribution, in terms of a GML function as

$$
f_{k}^{v}(t)=\operatorname{Pr}\left\{T_{k} \in d t\right\} / d t=\lambda^{k} t^{v k-1} E_{v, v k}^{k}\left(-\lambda t^{v}\right) .
$$

The corresponding distribution function can be obtained by integrating 2.19)

$$
\begin{aligned}
F_{k}^{v}(t) & =\operatorname{Pr}\left\{T_{k}<t\right\} \\
& =\lambda^{k} \int_{0}^{t} s^{v k-1} \sum_{j=0}^{\infty} \frac{(k-1+j) !\left(-\lambda s^{v}\right)^{j}}{j !(k-1) ! \Gamma(v j+v k)} d s \\
& =\frac{\lambda^{k} t^{v k}}{v} \sum_{j=0}^{\infty} \frac{(k-1+j) !\left(-\lambda t^{v}\right)^{j}}{j !(k-1) !(k+j) \Gamma(v j+v k)} \\
& =\lambda^{k} t^{v k} \sum_{j=0}^{\infty} \frac{(k-1+j) !\left(-\lambda t^{v}\right)^{j}}{j !(k-1) ! \Gamma(v j+v k+1)}=\lambda^{k} t^{v k} E_{v, v k+1}^{k}\left(-\lambda t^{v}\right) .
\end{aligned}
$$

We can check that (2.20) satisfies the following relationship

$$
\operatorname{Pr}\left\{T_{k}<t\right\}-\operatorname{Pr}\left\{T_{k+1}<t\right\}=p_{k}^{v}(t),
$$


for $p_{k}^{v}$ given in $(2.5)$. Indeed from (2.20) we can rewrite (2.21) as

$$
\begin{aligned}
& \lambda^{k} t^{v k} E_{v, v k+1}^{k}\left(-\lambda t^{v}\right)-\lambda^{k+1} t^{v(k+1)} E_{v, v(k+1)+1}^{k+1}\left(-\lambda t^{v}\right) \\
= & \lambda^{k} t^{v k} \sum_{j=0}^{\infty} \frac{(k-1+j) !\left(-\lambda t^{v}\right)^{j}}{j !(k-1) ! \Gamma(v j+v k+1)}-\lambda^{k+1} t^{v(k+1)} \sum_{j=0}^{\infty} \frac{(k+j) !\left(-\lambda t^{v}\right)^{j}}{j ! k ! \Gamma(v j+v k+v+1)} \\
= & {[\text { by putting } l=j+1 \text { in the second sum }] } \\
= & \lambda^{k} t^{v k} \sum_{j=0}^{\infty} \frac{(k-1+j) !\left(-\lambda t^{v}\right)^{j}}{j !(k-1) ! \Gamma(v j+v k+1)}+\lambda^{k} t^{v k} \sum_{l=1}^{\infty} \frac{(k+l-1) !\left(-\lambda t^{v}\right)^{l}}{(l-1) ! k ! \Gamma(v l+v k+1)} \\
= & \lambda^{k} t^{v k} \sum_{j=0}^{\infty} \frac{(k+j) !\left(-\lambda t^{v}\right)^{j}}{j ! k ! \Gamma(v j+v k+1)}=p_{k}^{v}(t) .
\end{aligned}
$$

Remark 2.3 As pointed out in [18] and [25], the density of the interarrival times (2.16) possess the following asymptotic behavior, for $t \rightarrow \infty$ :

$$
\begin{aligned}
\operatorname{Pr}\left\{\mathscr{U}_{j} \in d t\right\} / d t & =\lambda t^{v-1} E_{v, v}\left(-\lambda t^{v}\right)=-\frac{d}{d t} E_{v, 1}\left(-\lambda t^{v}\right) \\
& =\lambda^{1 / v} \frac{\sin (v \pi)}{\pi} \int_{0}^{+\infty} \frac{r^{v} e^{-\lambda^{1 / v} r t}}{r^{2 v}+2 r^{v} \cos (v \pi)+1} d r \\
& \sim \frac{\sin (v \pi)}{\pi} \frac{\Gamma(v+1)}{\lambda t^{v+1}}=\frac{v}{\lambda \Gamma(1-v) t^{v+1}},
\end{aligned}
$$

where the well-known expansion of the Mittag-Leffler function (given in (5.3) has been applied. The density (2.22) is characterized by fat tails (with polynomial, instead of exponential, decay) and, as a consequence, the mean waiting time is infinite.

For $t \rightarrow 0$ the density of the interarrival times displays the following behavior:

$$
\operatorname{Pr}\left\{\mathscr{U}_{j} \in d t\right\} / d t \sim \frac{\lambda t^{v-1}}{\Gamma(v)},
$$

which means that $\mathscr{U}_{j}$ takes small values with large probability. Therefore, by considering (2.22) and (2.23) together, we can draw the conclusion that the behavior of the density of the interarrival times differs from standard Poisson in that the intermediate values are assumed with smaller probability than in the exponential case.

Remark 2.4 We observe that also for the waiting-time density (2.19) we can find a link with the solution to the fractional diffusion equation (2.8). This can be shown by rewriting its Laplace transform (2.18) as

$$
\mathscr{L}\left\{f_{k}^{v}(t) ; s\right\}=\frac{\lambda^{k}}{\left(s^{v}+\lambda\right)^{k}}=\int_{0}^{+\infty} e^{-s^{v} t} \frac{\lambda^{k} t^{k-1}}{(k-1) !} e^{-\lambda t} d t .
$$

By recalling that

$$
e^{-s^{v} y / \lambda}=\int_{0}^{+\infty} e^{-s z} g_{v}\left(z ; \frac{y}{\lambda}\right) d z, \quad 0<v<1, y>0
$$


for the stable law $g_{v}(\cdot ; y)$ defined above, we get

$$
f_{k}^{v}(t)=\int_{0}^{+\infty} g_{v}\left(t ; \frac{y}{\lambda}\right) \frac{y^{k-1} e^{-y}}{(k-1) !} d y .
$$

Formula (2.25) permits us to conclude that $f_{k}^{v}(t)$ can be interpreted as the law of the stable random variable $S_{v}$ with a random scale parameter possessing an Erlang distribution.

\subsection{The probability generating function}

We consider now the equation governing the probability generating function, defined, for any $|u| \leq$ 1 , as

$$
G_{v}(u, t)=\sum_{k=0}^{\infty} u^{k} p_{k}^{v}(t)
$$

From (2.1) it is straightforward that it coincides with the solution to the fractional differential equation

$$
\frac{\partial^{v} G(u, t)}{\partial t^{v}}=\lambda(u-1) G(u, t), \quad 0<v \leq 1
$$

subject to the initial condition $G(u, 0)=1$. As already proved in [2] the Laplace transform of $G_{v}=$ $G_{v}(u, t)$ is given by

$$
\mathscr{L}\left\{G_{v}(u, t) ; s\right\}=\frac{s^{v-1}}{s^{v}-\lambda(u-1)}
$$

so that the probability generating function can be expressed as

$$
G_{v}(u, t)=E_{v, 1}\left(\lambda(u-1) t^{v}\right), \quad|u| \leq 1, t>0 .
$$

By considering (2.29) together with the previous results we get the following relationship, valid for the infinite sum of GML functions:

$$
\sum_{k=0}^{\infty}\left(\lambda u t^{v}\right)^{k} E_{v, v k+1}^{k+1}\left(-\lambda t^{v}\right)=E_{v, 1}\left(\lambda(u-1) t^{v}\right) .
$$

Formula (2.30) suggests a useful general relationship between the infinite sum of GML functions and the standard Mittag-Leffler function:

$$
\sum_{k=0}^{\infty}(u x)^{k} E_{v, v k+1}^{k+1}(-x)=E_{v, 1}(x(u-1)), \quad|u| \leq 1, \quad x>0 .
$$

By considering the derivatives of the probability generating function $(2.29)$ we can easily derive the factorial moments of $\mathscr{N}_{v}$ which read

$$
\mathbb{E}\left[\mathscr{N}_{v}(t)\left(\mathscr{N}_{v}(t)-1\right) \ldots\left(\mathscr{N}_{v}(t)-r+1\right)\right]=\frac{\left(\lambda t^{v}\right)^{r} r !}{\Gamma(v r+1)} .
$$

These are particularly useful in checking that $\mathscr{N}_{v}$ represents a Cox process with directing measure $\Lambda$. Indeed, as pointed out in [12], the factorial moments of a Cox process coincide with the ordinary 
moments of its directing measure. We show that this holds for $\mathscr{N}_{v}$, by using the contour integral representation of the inverse of Gamma function,

$$
\begin{aligned}
\mathbb{E}[\Lambda((0, t])]^{r} & =\int_{0}^{+\infty} y^{r} v_{2 v}(y, t) d y \\
& =\int_{0}^{+\infty} \frac{y^{r}}{\lambda t^{v}} W_{-v, 1-v}\left(-\frac{y}{\lambda t^{v}}\right) d y \\
& =\frac{1}{\lambda t^{v}} \frac{1}{2 \pi i} \int_{0}^{+\infty} y^{r} d y \int_{H a} \frac{e^{z-\frac{y t^{-v}}{\lambda} z^{v}}}{z^{1-v}} d z \\
& =\frac{\lambda^{r}}{2 \pi i} \int_{H a} \frac{e^{z}}{z^{1+v r}} d z \int_{0}^{+\infty} t^{v r} w^{r} e^{-w} d w \\
& =\frac{\lambda^{r} t^{v r}}{2 \pi i} \Gamma(r+1) \int_{H a} \frac{e^{z}}{z^{1+v r}} d z=\frac{\left(\lambda t^{v}\right)^{r} r !}{\Gamma(v r+1)},
\end{aligned}
$$

which coincides with (2.32).

For $r=1$ we can obtain from (2.32) the renewal function of the process $\mathscr{N}_{v}$, which reads

$$
\begin{aligned}
m_{v}(t) & =\mathbb{E} \mathscr{N}_{v}(t)=-\lambda t^{v}\left[\frac{d}{d \mu} E_{v, 1}\left(\lambda\left(e^{-\mu}-1\right) t^{v}\right)\right]_{\mu=0} \\
& =\lambda t^{v}\left[\frac{d}{d x} E_{v, 1}(x)\right]_{x=0}=\frac{\lambda t^{v}}{\Gamma(v+1)},
\end{aligned}
$$

and coincides with that obtained in [18], for $\lambda=1$. It is evident also from (2.33) that the mean waiting time (which is equal to $\lim _{t \rightarrow \infty} t / m_{v}(t)$ ) is infinite, since $v<1$.

\section{Second-type fractional recursive differential equation}

\subsection{The solution}

In this section we generalize the results obtained so far by introducing in the fractional recursive differential equation an additional time-fractional derivative. We show that some properties of the first model of fractional Poisson process are still valid: the solutions represent, for $k \geq 0$, a proper probability distribution and the corresponding process is again a renewal process. Moreover the density of the interarrival times displays the same asymptotic behavior of the previous model.

We consider the following recursive differential equation

$$
\frac{d^{2 v} p_{k}}{d t^{2 v}}+2 \lambda \frac{d^{v} p_{k}}{d t^{v}}=-\lambda^{2}\left(p_{k}-p_{k-1}\right), \quad k \geq 0,
$$

where $v \in(0,1]$, subject to the initial conditions

$$
\begin{aligned}
& p_{k}(0)=\left\{\begin{array}{ll}
1 & k=0 \\
0 & k \geq 1
\end{array}, \quad \text { for } 0<v \leq 1\right. \\
& p_{k}^{\prime}(0)=0, \quad k \geq 0, \quad \text { for } \frac{1}{2}<v \leq 1
\end{aligned}
$$


and $p_{-1}(t)=0$. In the following theorem we derive the solution to $(3.1)-(3.2)$, which can be still expressed in terms of GML functions.

Theorem 3.1 The solution $\widehat{p}_{k}^{v}(t)$, for $k=0,1, \ldots$ and $t \geq 0$, of the Cauchy problem (3.1)-(3.2) is given by

$$
\hat{p}_{k}^{v}(t)=\lambda^{2 k} t^{2 k v} E_{v, 2 k v+1}^{2 k+1}\left(-\lambda t^{v}\right)+\lambda^{2 k+1} t^{(2 k+1) v} E_{v,(2 k+1) v+1}^{2 k+2}\left(-\lambda t^{v}\right), \quad k \geq 0, t>0 .
$$

Proof Following the lines of the proof of Theorem 2.1, we take the Laplace transform of equation (3.1) together with the conditions (3.2), thus obtaining the following recursive formula, for $k \geq 1$

$$
\mathscr{L}\left\{\widehat{p}_{k}^{v}(t) ; s\right\}=\frac{\lambda^{2}}{s^{2 v}+2 \lambda s^{v}+\lambda^{2}} \mathscr{L}\left\{\widehat{p}_{k-1}^{v}(t) ; s\right\}=\frac{\lambda^{2}}{\left(s^{v}+\lambda\right)^{2}} \mathscr{L}\left\{\widehat{p}_{k-1}^{v}(t) ; s\right\},
$$

while, for $k=0$, we get

$$
\mathscr{L}\left\{\widehat{p}_{0}^{v}(t) ; s\right\}=\frac{s^{2 v-1}+2 \lambda s^{v-1}}{s^{2 v}+2 \lambda s^{v}+\lambda^{2}} .
$$

Therefore the Laplace transform of the solution reads

$$
\mathscr{L}\left\{\widehat{p}_{k}^{v}(t) ; s\right\}=\frac{\lambda^{2 k} s^{2 v-1}+2 \lambda^{2 k+1} s^{v-1}}{\left(s^{v}+\lambda\right)^{2 k+2}} .
$$

We can invert (3.4) by using (2.7) with $\delta=2 k+2, \beta=v$ and $\gamma=2 k v+1$ or $\gamma=(2 k+1) v+1$, thus obtaining the following expression

$$
\widehat{p}_{k}^{v}(t)=\lambda^{2 k} t^{2 v k} E_{v, 2 k v+1}^{2 k+2}\left(-\lambda t^{v}\right)+2 \lambda^{2 k+1} t^{(2 k+1) v} E_{v,(2 k+1) v+1}^{2 k+2}\left(-\lambda t^{v}\right) .
$$

We prove now the following general formula holding for a sum of GML functions:

$$
x^{n} E_{v, n v+z}^{m}(-x)+x^{n+1} E_{v,(n+1) v+z}^{m}(-x)=x^{n} E_{v, n v+z}^{m-1}(-x), \quad n, m>0, z \geq 0, x>0,
$$

which can be checked by rewriting the 1.h.s. as follows:

$$
\begin{aligned}
& \frac{x^{n}}{(m-1) !} \sum_{j=0}^{\infty} \frac{(m-1+j) !(-x)^{j}}{j ! \Gamma(v j+n v+z)}-\frac{x^{n}}{(m-1) !} \sum_{j=0}^{\infty} \frac{(m-1+j) !(-x)^{j+1}}{j ! \Gamma(v j+(n+1) v+z)} \\
= & \frac{x^{n}}{(m-1) !} \sum_{j=0}^{\infty} \frac{(m-1+j) !(-x)^{j}}{j ! \Gamma(v j+n v+z)}-\frac{x^{n}}{(m-1) !} \sum_{l=1}^{\infty} \frac{(m+l-2) !(-x)^{l}}{(l-1) ! \Gamma(v l+n v+z)} \\
= & \frac{x^{n}}{(m-1) !} \sum_{l=1}^{\infty} \frac{(m+l-2) !(-x)^{l}}{(l-1) ! \Gamma(v l+n v+z)}\left[\frac{m-1+l}{l}-1\right]+\frac{x^{n}}{\Gamma(n v+z)} \\
= & \frac{x^{n}}{(m-2) !} \sum_{l=1}^{\infty} \frac{(m+l-2) !(-x)^{l}}{l ! \Gamma(v l+n v+z)}+\frac{x^{n}}{\Gamma(n v+z)}=x^{n} E_{v, n v+z}^{m-1}(-x)
\end{aligned}
$$

For $m=2 k+2, z=1, x=\lambda t^{v}$ and $n=2 k$ formula (3.6) gives the following identity:

$$
\lambda^{2 k} t^{2 v k} E_{v, 2 k v+1}^{2 k+2}\left(-\lambda t^{v}\right)+\lambda^{2 k+1} t^{(2 k+1) v} E_{v,(2 k+1) v+1}^{2 k+2}\left(-\lambda t^{v}\right)=\lambda^{2 k} t^{2 v k} E_{v, 2 k v+1}^{2 k+1}\left(-\lambda t^{v}\right),
$$


which coincides with the first term in (3.3).

It remains to check only that the initial conditions in (3.2) hold: the first one is clearly satisfied since, for $k=0$, we have that

$$
\left.\widehat{p}_{0}^{v}(t)\right|_{t=0}=\sum_{r=0}^{\infty} \frac{(-\lambda)^{r} t^{v r}}{\Gamma(v r+1)}+\left.\lambda \sum_{r=0}^{\infty} \frac{(r+1)(-\lambda)^{r} t^{v(r+1)}}{\Gamma(v r+v+1)}\right|_{t=0}=1
$$

and, for $k \geq 1$,

$$
\widehat{p}_{k}^{v}(t)=\frac{\lambda^{2 k}}{(2 k) !} \sum_{r=0}^{\infty} \frac{(2 k+r) !(-\lambda)^{r} t^{v(2 k+r)}}{r ! \Gamma(v r+2 k v+1)}+\frac{\lambda^{2 k+1}}{(2 k+1) !} \sum_{r=0}^{\infty} \frac{(2 k+r+1) !(-\lambda)^{r} t^{v(2 k+r+1)}}{r ! \Gamma(v r+2 k v+v+1)},
$$

which vanishes for $t=0$. The second condition in $(3.2)$ is immediately verified for $k \geq 1$, since it is

$$
\frac{d}{d t} \widehat{p}_{k}^{v}(t)=\frac{\lambda^{2 k}}{(2 k) !} \sum_{r=1}^{\infty} \frac{(2 k+r) !(-\lambda)^{r} t^{v(2 k+r)-1}}{r ! \Gamma(v r+2 k v)}+\frac{\lambda^{2 k+1}}{(2 k+1) !} \sum_{r=0}^{\infty} \frac{(2 k+r+1) !(-\lambda)^{r} t^{v(2 k+r+1)-1}}{r ! \Gamma(v r+2 k v+v)}
$$

which for $t=0$ vanishes in the interval $\frac{1}{2}<v \leq 1$. Then we check that this happens also for $k=0$ : indeed in this case (3.7) reduces to

$$
\begin{aligned}
\left.\frac{d}{d t} \widehat{p}_{0}^{v}(t)\right|_{t=0} & =\sum_{r=1}^{\infty} \frac{(-\lambda)^{r} t^{v r-1}}{\Gamma(v r)}+\left.\lambda \sum_{r=0}^{\infty} \frac{(r+1)^{2}(-\lambda)^{r} t^{v(r+1)-1}}{\Gamma(v r+v)}\right|_{t=0} \\
& =\sum_{r=2}^{\infty} \frac{(-\lambda)^{r} t^{v r-1}}{\Gamma(v r)}-\frac{\lambda t^{v-1}}{\Gamma(v)}+\lambda \sum_{r=1}^{\infty} \frac{(r+1)^{2}(-\lambda)^{r} t^{v(r+1)-1}}{\Gamma(v r+v)}+\left.\frac{\lambda t^{v-1}}{\Gamma(v)}\right|_{t=0}=0 .
\end{aligned}
$$

Remark 3.1 The solution (3.3) can be expressed in terms of the solution (2.5) of the first model as follows

$$
\widehat{p}_{k}^{v}(t)=p_{2 k}^{v}(t)+p_{2 k+1}^{v}(t) .
$$

Therefore it can be interpreted, for $k=0,1,2, \ldots$, as the probability distribution $\operatorname{Pr}\left\{\widehat{\mathscr{N}_{v}}(t)=k\right\}$ for a process $\widehat{\mathbb{N}_{v}}$. Indeed, by (3.8), we get

$$
\operatorname{Pr}\left\{\widehat{\mathscr{N}_{v}}(t)=k\right\}=\operatorname{Pr}\left\{\mathscr{N}_{v}(t)=2 k\right\}+\operatorname{Pr}\left\{\mathscr{N}_{v}(t)=2 k+1\right\},
$$

so that it is immediate that (3.3) sums up to unity.

Moreover the relationship (3.8) shows that the process governed by the second-type equation can be seen as a first-type fractional process, which jumps upward at even-order events $A_{2 k}$ while the probability of the successive odd-indexed events $A_{2 k+1}$ is added to that of $A_{2 k}$.

A direct check that expression (3.8) is the solution to equation (3.1), subject to the initial conditions (3.2), can be carried out by using the form of $p_{k}^{v}$ appearing in formula (2.10) of [2] which is more suitable to this aim. Indeed, by substituting it into (3.8), the latter can be rewritten as

$$
\widehat{p}_{k}^{v}(t)=\sum_{r=2 k}^{\infty}\left(\begin{array}{c}
r \\
2 k
\end{array}\right) \frac{\left(-\lambda t^{v}\right)^{r}}{\Gamma(v r+1)}-\sum_{r=2 k+1}^{\infty}\left(\begin{array}{c}
r \\
2 k+1
\end{array}\right) \frac{\left(-\lambda t^{v}\right)^{r}}{\Gamma(v r+1)} .
$$


By taking the fractional derivatives of (3.10) and performing some manipulations, we get that

$$
\begin{aligned}
& \frac{d^{2 v}}{d t^{2 v}} \widehat{p}_{k}^{v}+2 \lambda \frac{d^{v}}{d t^{v}} \widehat{p}_{k}^{v} \\
= & \sum_{r=2 k-2}^{\infty}\left(\begin{array}{c}
r+2 \\
2 k
\end{array}\right) A_{r}-\sum_{r=2 k-1}^{\infty}\left(\begin{array}{c}
r+2 \\
2 k+1
\end{array}\right) A_{r}+ \\
& -2 \sum_{r=2 k-1}^{\infty}\left(\begin{array}{c}
r+1 \\
2 k
\end{array}\right) A_{r}+2 \sum_{r=2 k}^{\infty}\left(\begin{array}{c}
r+1 \\
2 k+1
\end{array}\right) A_{r},
\end{aligned}
$$

where $A_{r}=\frac{(-1)^{r} \lambda^{r+2} t^{v r}}{\Gamma(v r+1)}$. By means of some combinatorial results it can be checked that 3.11) is equal to

$$
\begin{aligned}
& -\lambda^{2}\left(\widehat{p}_{k}^{v}-\widehat{p}_{k-1}^{v}\right) \\
= & -\lambda^{2}\left(p_{2 k}^{v}+p_{2 k+1}^{v}-p_{2 k-2}^{v}-p_{2 k-1}^{v}\right) \\
= & -\sum_{r=2 k}^{\infty}\left(\begin{array}{c}
r \\
2 k
\end{array}\right) A_{r}+\sum_{r=2 k+1}^{\infty}\left(\begin{array}{c}
r \\
2 k+1
\end{array}\right) A_{r}+ \\
& +\sum_{r=2 k-2}^{\infty}\left(\begin{array}{c}
r \\
2 k-2
\end{array}\right) A_{r}-\sum_{r=2 k-1}^{\infty}\left(\begin{array}{c}
r \\
2 k-1
\end{array}\right) A_{r},
\end{aligned}
$$

(see [3], for a detailed proof) and thus (3.10) satisfies equation (3.1).

\subsection{The probability generating function}

As we did for the first model we evaluate the probability generating function and we show that it coincides with the solution to a fractional equation which arises in the study of the fractional telegraph process (see [20]).

Theorem 3.2 The probability generating function $\widehat{G}_{v}(u, t)=\sum_{k=0}^{\infty} u^{k} \widehat{p}_{k}^{v}(t),|u| \leq 1$, coincides with the solution to the following fractional differential equation

$$
\frac{\partial^{2 v} G(u, t)}{\partial t^{2 v}}+2 \lambda \frac{\partial^{v} G(u, t)}{\partial t^{v}}=\lambda^{2}(u-1) G(u, t), \quad 0<v \leq 1
$$

subject to the initial condition $G(u, 0)=1$ and the additional condition $G_{t}(u, 0)=0$ for $1 / 2<v<1$. The explicit expression is given by

$$
\widehat{G}_{v}(u, t)=\frac{\sqrt{u}+1}{2 \sqrt{u}} E_{v, 1}\left(-\lambda(1-\sqrt{u}) t^{v}\right)+\frac{\sqrt{u}-1}{2 \sqrt{u}} E_{v, 1}\left(-\lambda(1+\sqrt{u}) t^{v}\right) .
$$

Proof By applying the Laplace transform to 3.13 , we get

$$
\left(s^{2 v}+2 \lambda s^{v}\right) \mathscr{L}\left(\widehat{G}_{v}(u, t) ; s\right)+\left(s^{2 v-1}+2 \lambda s^{v-1}\right)=\lambda^{2}(u-1) \mathscr{L}\left(\widehat{G}_{v}(u, t) ; s\right)
$$

and then

$$
\mathscr{L}\left(\widehat{G}_{v}(u, t) ; s\right)=\frac{s^{2 v-1}+2 \lambda s^{v-1}}{s^{2 v}+2 \lambda s^{v}+\lambda^{2}(1-u)}
$$


We can recognize in (3.13) the fractional equation satisfied by the characteristic function of the fractional telegraph process studied in [20] (see formula (2.3a) with $c^{2} \beta^{2}=\lambda^{2}(1-u)$ ) and thus the Laplace transform (3.15) coincides with formula (2.6) therein. By applying the result of Theorem 2.1 of the cited paper, we obtain the inverse Laplace transform of (3.15) as given in (3.14).

Remark 3.2 As an alternative proof of the Theorem 3.1, we can show that the series expansion of (3.14) coincides with $\sum_{k=0}^{\infty} u^{k} \widehat{p}_{k}^{v}(t)$ for $\widehat{p}_{k}^{v}(t)$ given in (3.3):

$$
\begin{aligned}
& \widehat{G}_{v}(u, t) \\
& =\frac{\sqrt{u}+1}{2 \sqrt{u}} \sum_{j=0}^{\infty} \frac{\left(-\lambda(1-\sqrt{u}) t^{v}\right)^{j}}{\Gamma(v j+1)}+\frac{\sqrt{u}-1}{2 \sqrt{u}} \sum_{j=0}^{\infty} \frac{\left(-\lambda(1+\sqrt{u}) t^{v}\right)^{j}}{\Gamma(v j+1)} \\
& =\frac{\sqrt{u}+1}{2 \sqrt{u}} \sum_{j=0}^{\infty} \frac{\left(\lambda t^{v}\right)^{j}}{\Gamma(v j+1)} \sum_{k=0}^{j}\left(\begin{array}{l}
j \\
k
\end{array}\right)(\sqrt{u})^{k}(-1)^{j-k}+\frac{\sqrt{u}-1}{2 \sqrt{u}} \sum_{j=0}^{\infty} \frac{\left(-\lambda t^{v}\right)^{j}}{\Gamma(v j+1)} \sum_{k=0}^{j}\left(\begin{array}{l}
j \\
k
\end{array}\right)(\sqrt{u})^{k} \\
& =\frac{\sqrt{u}+1}{2 \sqrt{u}} \sum_{k=0}^{\infty}(-\sqrt{u})^{k} \sum_{j=k}^{\infty} \frac{\left(-\lambda t^{v}\right)^{j}}{\Gamma(v j+1)}\left(\begin{array}{l}
j \\
k
\end{array}\right)+\frac{\sqrt{u}-1}{2 \sqrt{u}} \sum_{k=0}^{\infty}(\sqrt{u})^{k} \sum_{j=k}^{\infty} \frac{\left(-\lambda t^{v}\right)^{j}}{\Gamma(v j+1)}\left(\begin{array}{l}
j \\
k
\end{array}\right) \\
& =\frac{\sqrt{u}+1}{2 \sqrt{u}} \sum_{k=0}^{\infty}\left(\sqrt{u} \lambda t^{v}\right)^{k} \sum_{l=0}^{\infty} \frac{\left(-\lambda t^{v}\right)^{l}}{\Gamma(v l+v k+1)}\left(\begin{array}{c}
l+k \\
k
\end{array}\right)+ \\
& +\frac{\sqrt{u}-1}{2 \sqrt{u}} \sum_{k=0}^{\infty}\left(-\sqrt{u} \lambda t^{v}\right)^{k} \sum_{l=0}^{\infty} \frac{\left(-\lambda t^{v}\right)^{l}}{\Gamma(v l+v k+1)}\left(\begin{array}{c}
l+k \\
k
\end{array}\right) \\
& =\frac{\sqrt{u}+1}{2 \sqrt{u}} \sum_{k=0}^{\infty}\left(\sqrt{u} \lambda t^{v}\right)^{k} E_{v, v k+1}^{k+1}\left(-\lambda t^{v}\right)+\frac{\sqrt{u}-1}{2 \sqrt{u}} \sum_{k=0}^{\infty}\left(-\sqrt{u} \lambda t^{v}\right)^{k} E_{v, v k+1}^{k+1}\left(-\lambda t^{v}\right) \\
& =\frac{\sqrt{u}+1}{2 \sqrt{u}} \sum_{m=0}^{\infty}\left(\sqrt{u} \lambda t^{v}\right)^{2 m} E_{v, 2 m v+1}^{2 m+1}\left(-\lambda t^{v}\right)+\frac{\sqrt{u}+1}{2 \sqrt{u}} \sum_{m=0}^{\infty}\left(\sqrt{u} \lambda t^{v}\right)^{2 m+1} E_{v,(2 m+1) v+1}^{2 m+2}\left(-\lambda t^{v}\right)+ \\
& +\frac{\sqrt{u}-1}{2 \sqrt{u}} \sum_{m=0}^{\infty}\left(\sqrt{u} \lambda t^{v}\right)^{2 m} E_{v, 2 m v+1}^{2 m+1}\left(-\lambda t^{v}\right)+\frac{\sqrt{u}-1}{2 \sqrt{u}} \sum_{m=0}^{\infty}\left(-\sqrt{u} \lambda t^{v}\right)^{2 m+1} E_{v,(2 m+1) v+1}^{2 m+2}\left(-\lambda t^{v}\right) \\
& =\sum_{m=0}^{\infty} u^{m}\left[\left(\lambda t^{v}\right)^{2 m} E_{v, 2 m v+1}^{2 m+1}\left(-\lambda t^{v}\right)+\left(\lambda t^{v}\right)^{2 m+1} E_{v,(2 m+1) v+1}^{2 m+2}\left(-\lambda t^{v}\right)\right] \text {. }
\end{aligned}
$$

Since, for $u=1$, formula (3.14) reduces to one, it is proved that $\sum_{k=0}^{\infty} \widehat{p}_{k}^{v}(t)=1$.

\subsection{Properties of the corresponding process}

We can prove that $\widehat{N_{v}}(t), t>0$ represents a renewal process, by showing that, also for this model, the required relationship between $\widehat{p}_{k}^{v}(t)$ and distribution function of the waiting time $\widehat{T}_{k}$ of the $k$-th event holds:

$$
\widehat{p}_{k}^{v}(t)=\operatorname{Pr}\left\{\widehat{T}_{k}<t\right\}-\operatorname{Pr}\left\{\widehat{T}_{k+1}<t\right\}
$$

where

$$
\widehat{T}_{k}=\inf \left\{t>0: \widehat{\mathscr{N}_{v}}(t)=k\right\}
$$


or alternatively for the Laplace transform of 3.16

$$
\mathscr{L}\left\{\widehat{p}_{k}^{v}(t) ; s\right\}=\frac{1}{s} \mathscr{L}\left\{\widehat{f}_{k}^{v}(t) ; s\right\}-\frac{1}{s} \mathscr{L}\left\{\widehat{f}_{k+1}^{v}(t) ; s\right\} .
$$

In view of relationship $(3.8)$, we can infer that each interarrival time $\widehat{\mathscr{U}_{j}}$ is distributed as the sum of two independent interarrival times $\mathscr{U}_{j}$ of the first model and therefore from (2.17) we have that

$$
\begin{aligned}
\mathscr{L}\left\{\widehat{f}_{1}^{v}(t) ; s\right\} & =\int_{0}^{\infty} e^{-s t} \operatorname{Pr}\left\{\widehat{\mathscr{U}}_{j} \in d t\right\}=\left[\int_{0}^{\infty} e^{-s t} \operatorname{Pr}\left\{\mathscr{U}_{j} \in d t\right\}\right]^{2} \\
& =\frac{\lambda^{2}}{\left(s^{v}+\lambda\right)^{2}}
\end{aligned}
$$

and therefore

$$
\mathscr{L}\left\{\widehat{f}_{k}^{v}(t) ; s\right\}=\frac{\lambda^{2 k}}{\left(s^{v}+\lambda\right)^{2 k}} .
$$

The relationship (3.18) can be checked directly as follows

$$
\int_{0}^{t} f_{1}^{v}(s) f_{1}^{v}(t-s) d s=\lambda^{2} t^{2 v-1} E_{v, 2 v}^{2}\left(-\lambda t^{v}\right)=\widehat{f}_{1}^{v}(t)
$$

(see [3] for details). By applying again (2.7) for $\beta=v, \gamma=2 v k$ and $\omega=-\lambda$ we invert (3.19) and obtain

$$
\widehat{f}_{k}^{v}(t)=\lambda^{2 k} t^{2 v k-1} E_{v, 2 v k}^{2 k}\left(-\lambda t^{v}\right) .
$$

Moreover from (3.18) or (3.21) we easily get

$$
\widehat{f}_{1}^{v}(t)=\lambda^{2} t^{2 v-1} E_{v, 2 v}^{2}\left(-\lambda t^{v}\right)
$$

Therefore, in this case, both the waiting-time of the $k$-th event and the interarrival times possess distributions which are expressed in terms of GML functions.

By performing steps similar to those of Remark 4.1 of [2], we can conclude from the previous results that the Laplace transform (3.19), together with (3.4) satisfy the renewal property (3.17).

Remark 3.3 We evaluate now the asymptotic behavior of the interarrival-time density (3.22), as follows:

$$
\begin{aligned}
\operatorname{Pr}\left\{\widehat{\mathscr{U}_{j}} \in d t\right\} / d t & =\lambda^{2} t^{2 v-1} \sum_{j=0}^{\infty} \frac{\left(-\lambda t^{v}\right)^{j}(j+1) !}{j ! \Gamma(v j+2 v)} \\
& =\lambda^{2} t^{2 v-1} \sum_{l=0}^{\infty} \frac{l\left(-\lambda t^{v}\right)^{l-1}}{\Gamma(v l+v)}=-\frac{\lambda t^{v}}{v} \frac{d}{d t} E_{v, v}\left(-\lambda t^{v}\right) \\
& =\frac{1-v}{v} \frac{d}{d t} E_{v, 1}\left(-\lambda t^{v}\right)+\frac{t}{v} \frac{d^{2}}{d t^{2}} E_{v, 1}\left(-\lambda t^{v}\right) .
\end{aligned}
$$


By applying (2.22) and (5.3), we finally get

$$
\begin{aligned}
\operatorname{Pr}\left\{\widehat{\mathscr{U}_{j}} \in d t\right\} / d t= & \frac{v-1}{v} \lambda^{1 / v} \frac{\sin (v \pi)}{\pi} \int_{0}^{+\infty} \frac{r^{v} e^{-\lambda^{1 / v} r t}}{r^{2 v}+2 r^{v} \cos (v \pi)+1} d r+ \\
& +\frac{\lambda^{2 / v} t}{v} \frac{\sin (v \pi)}{\pi} \int_{0}^{+\infty} \frac{r^{v+1} e^{-\lambda^{1 / v} r t}}{r^{2 v}+2 r^{v} \cos (v \pi)+1} d r \\
\sim & \frac{\sin (v \pi)}{\pi} \frac{2 \Gamma(v+1)}{\lambda t^{v+1}}=\frac{2 v}{\lambda \Gamma(1-v) t^{v+1}}
\end{aligned}
$$

If we compare (3.24) with the analogous result (2.22) obtained for the first model, we can conclude that the interarrival-times density displays the same asymptotic behavior, with the power law decay (3.24), so that again the mean waiting time is infinite.

For $t \rightarrow 0$, instead of (2.23), we get in this case

$$
\operatorname{Pr}\left\{\widehat{\mathscr{U}_{j}} \in d t\right\} / d t \sim \frac{\lambda^{2} t^{2 v-1}}{\Gamma(2 v)} .
$$

The behavior near the origin of the density of the interarrival times $\widehat{\mathscr{U}_{j}}$ has a different structure for $v<1 / 2$ (tends to infinity) and $v \in(1 / 2,1]$ (vanishes for $t \rightarrow 0^{+}$). For $v=\frac{1}{2}$ we have instead that $\operatorname{Pr}\left\{\widehat{\mathscr{U}_{j}} \in d t\right\}$ is constant for $t=0$ and equal to $\lambda^{2}$.

From all the previous considerations, we can conclude that $\widehat{N_{v}}$ is a renewal process and the corresponding renewal function is given by

$$
\begin{aligned}
\widehat{m}_{v}(t) & =\mathbb{E} \widehat{\mathscr{N}_{v}}(t)=\lambda^{2} t^{2 v} E_{v, 2 v+1}\left(-2 \lambda t^{v}\right) \\
& =\frac{\lambda t^{v}}{2 \Gamma(v+1)}-\frac{\lambda t^{v}}{2} E_{v, v+1}\left(-2 \lambda t^{v}\right) .
\end{aligned}
$$

Formula (3.25) can be obtained by taking the first derivative of (3.15) (for $u=1$ ):

$$
\left.\frac{\partial}{\partial u} \mathscr{L}\left(\widehat{G}_{v}(u, t) ; s\right)\right|_{u=1}=\frac{\lambda^{2} s^{-v-1}}{s^{v}+2 \lambda} .
$$

By applying (2.7) for $\beta=v, \delta=1$ and $\gamma=2 v+1$, we get (3.25).

Remark 3.4 By comparing the second form of (3.25) with (2.33), we can note that the following relationship between the renewal functions of the two models holds:

$$
\widehat{m}_{v}(t)=\frac{m_{v}(t)}{2}-\frac{\lambda t^{v}}{2} E_{v, v+1}\left(-2 \lambda t^{v}\right)
$$


This can be alternatively proved by applying (3.8) as follows:

$$
\begin{aligned}
\widehat{m}_{v}(t) & =\sum_{k=0}^{\infty} k \widehat{p}_{k}^{v}(t)=\sum_{k=0}^{\infty} k p_{2 k}^{v}(t)+\sum_{k=0}^{\infty} k p_{2 k+1}^{v}(t) \\
& =\frac{1}{2} \sum_{k=0}^{\infty}(2 k) p_{2 k}^{v}(t)+\frac{1}{2} \sum_{k=0}^{\infty}(2 k+1) p_{2 k+1}^{v}(t)-\frac{1}{2} \sum_{k=0}^{\infty} p_{2 k+1}^{v}(t) \\
& =\frac{1}{2} \sum_{j=0}^{\infty} j p_{j}^{v}(t)-\frac{1}{2} \sum_{k=0}^{\infty} p_{2 k+1}^{v}(t) \\
& =\frac{m_{v}(t)}{2}-\frac{1}{2} \sum_{k=0}^{\infty} p_{2 k+1}^{v}(t) .
\end{aligned}
$$

The last term in (3.27), which coincides with the sum of the probabilities of an odd number of events of the first model, can be evaluated as follows:

$$
\sum_{k=0}^{\infty} p_{2 k+1}^{v}(t)=\sum_{k=0}^{\infty} \lambda^{2 k+1} t^{(2 k+1) v} E_{v, v(2 k+1)+1}^{2 k+2}\left(-\lambda t^{v}\right)=\lambda t^{v} E_{v, v+1}\left(-2 \lambda t^{v}\right)
$$

as can be checked by resorting to the Laplace transform:

$$
\sum_{k=0}^{\infty} \frac{\lambda^{2 k+1} s^{v-1}}{\left(s^{v}+\lambda\right)^{2 k+2}}=\frac{\lambda}{s\left(s^{v}+2 \lambda\right)}=\mathscr{L}\left\{\lambda t^{v} E_{v, v+1}\left(-2 \lambda t^{v}\right) ; s\right\}
$$

Formula (3.26) confirms that the mean waiting time is infinite (as we have noticed in Remark 3.4): indeed it is

$$
\lim _{t \rightarrow \infty} \frac{\widehat{m}_{v}(t)}{t}=\lim _{t \rightarrow \infty} \frac{m_{v}(t)}{2 t}-\lim _{t \rightarrow \infty} \frac{\lambda t^{v-1}}{2} E_{v, v+1}\left(-2 \lambda t^{v}\right)=0
$$

where the second limit can be evaluated by the following considerations:

$$
\frac{\lambda t^{v-1}}{2} E_{v, v+1}\left(-2 \lambda t^{v}\right)=\frac{1}{4 t}\left[1-E_{v, 1}\left(-2 \lambda t^{v}\right)\right] \sim \frac{1}{4 t}\left[1-\frac{\sin (\pi v)}{\pi} \frac{\Gamma(v)}{2 \lambda t^{v}}\right]
$$

(see (5.3) in the Appendix).

\subsection{The special case $v=1$}

We consider now the previous results in the special case $v=1$. Equation (3.1) reduces in this case to the second-order equation:

$$
\frac{d^{2} \widehat{p}_{k}}{d t^{2}}+2 \lambda \frac{d \widehat{p}_{k}}{d t}=-\lambda^{2}\left(\widehat{p}_{k}-\widehat{p}_{k-1}\right), \quad k \geq 0
$$

and the corresponding solution (3.3) is given by

$$
\widehat{p}_{k}(t)=\frac{(\lambda t)^{2 k}}{(2 k) !} e^{-\lambda t}+\frac{(\lambda t)^{2 k+1}}{(2 k+1) !} e^{-\lambda t}, \quad k \geq 0 .
$$


It is easy to check directly that (3.29) solves (3.28) with initial condition

$$
\begin{aligned}
& \widehat{p}_{k}(0)= \begin{cases}1 & k=0 \\
0 & k \geq 1\end{cases} \\
& \widehat{p}_{k}^{\prime}(0)=0, \quad k \geq 0
\end{aligned}
$$

and $\widehat{p}_{-1}(t)=0$.

Formula (3.29) agrees with the relationship (3.8) given in Remark 3.1, which in this case we can write as follows

$$
\widehat{p}_{k}(t)=p_{2 k}(t)+p_{2 k+1}(t) .
$$

Therefore it can be interpreted, for $k=0,1,2, \ldots$, as the probability distribution $\operatorname{Pr}\{\widehat{N}(t)=k\}$ for a "second-order process" $\widehat{N}(t), t>0$ linked to the standard Poisson process $N$ by the following relationship:

$$
\operatorname{Pr}\{\widehat{N}(t)=k\}=\operatorname{Pr}\{N(t)=2 k\}+\operatorname{Pr}\{N(t)=2 k+1\} .
$$

From (3.21) and (3.22) we can easily see that for this process the densities of the interarrival times and of the $k$-th event waiting time are given respectively by

$$
\widehat{f}_{1}(t)=\lambda^{2} t e^{-\lambda t}
$$

and

$$
\widehat{f}_{k}(t)=\frac{\lambda^{2 k} t^{2 k-1}}{(2 k-1) !} e^{-\lambda t}
$$

Therefore, in this case, the random variable $\widehat{T}_{j}$, representing the instant of the $j$-th event, is distributed as $\operatorname{Gamma}(\lambda, 2 j)$.

We derive equation (3.29) in an alternative way, which is similar to the construction of the standard Poisson process, by considering the relationships (3.9) or, equivalently, the property of the interarrival times described by 3.20 . We can write that

$$
\widehat{T}_{j} \stackrel{\text { law }}{=} T_{2 j}
$$

where

$$
T_{j}=\inf \{t>0: N(t)=j\}
$$

which represents the time of the $j$-th event of $N(t)$.

Let us consider now the following intervals

$$
\begin{aligned}
A & =\left[T_{2 k-2}, T_{2 k-1}\right) \\
B & =\left[T_{2 k-1}, T_{2 k}\right) \\
C & =\left[T_{2 k}, T_{2 k+1}\right) \\
D & =\left[T_{2 k+1}, T_{2 k+2}\right)
\end{aligned}
$$

so that $\left[T_{2 k-2}, T_{2 k+2}\right)=A \cup B \cup C \cup D$ (see Fig.1).

We evaluate the following probability, by stopping the approximation at the second-order terms: 


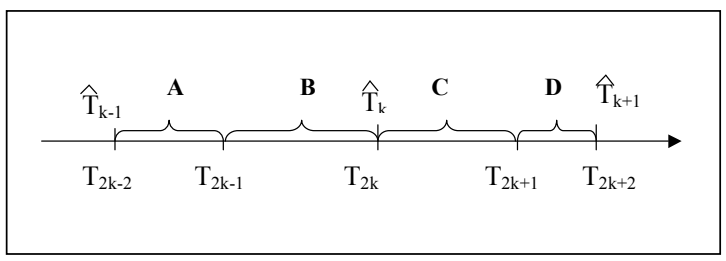

Figure 1: The interval $\left[T_{2 k-2}, T_{2 k+2}\right)$

$$
\begin{aligned}
& \widehat{p}_{k}(t+2 \Delta t) \\
& \simeq \quad 2 \operatorname{Pr}(\widehat{N}(t)=k-1, t \in A) \frac{\lambda^{2}(\Delta t)^{2}}{2}\left(1-\frac{\lambda^{2}(\Delta t)^{2}}{2}\right)+ \\
& +\operatorname{Pr}(\widehat{N}(t)=k-1, t \in A)\left(\lambda \Delta t-\lambda^{2}(\Delta t)^{2}\right)^{2}+ \\
& +2 \operatorname{Pr}(\widehat{N}(t)=k-1, t \in B)\left(\lambda \Delta t-\lambda^{2}(\Delta t)^{2}\right)\left(1-\lambda \Delta t+\frac{\lambda^{2}(\Delta t)^{2}}{2}\right)+ \\
& +2 \operatorname{Pr}(\widehat{N}(t)=k-1, t \in B) \frac{\lambda^{2}(\Delta t)^{2}}{2}\left(1-\lambda \Delta t+\frac{\lambda^{2}(\Delta t)^{2}}{2}\right)+ \\
& +\operatorname{Pr}(\widehat{N}(t)=k-1, t \in B)\left(\lambda \Delta t-\lambda^{2}(\Delta t)^{2}\right)^{2}+ \\
& +\operatorname{Pr}(\widehat{N}(t)=k, t \in C)\left(1-\lambda \Delta t+\frac{\lambda^{2}(\Delta t)^{2}}{2}\right)^{2}+ \\
& +2 \operatorname{Pr}(\widehat{N}(t)=k, t \in C)\left(1-\lambda \Delta t+\frac{\lambda^{2}(\Delta t)^{2}}{2}\right)^{2}\left(\lambda \Delta t-\lambda^{2}(\Delta t)^{2}\right)+ \\
& +\operatorname{Pr}(\widehat{N}(t)=k, t \in D)\left(1-\lambda \Delta t+\frac{\lambda^{2}(\Delta t)^{2}}{2}\right)^{2}+o\left((\Delta t)^{2}\right)
\end{aligned}
$$

where we used the following well-known approximations valid for the standard Poisson process:

$$
\begin{aligned}
\operatorname{Pr}(0 \text { Poisson events in } \Delta t) & =e^{-\lambda \Delta t} \simeq 1-\lambda \Delta t+\frac{\lambda^{2}(\Delta t)^{2}}{2}+o\left((\Delta t)^{2}\right) \\
\operatorname{Pr}(1 \text { Poisson event in } \Delta t) & =\lambda \Delta t e^{-\lambda \Delta t} \simeq \lambda \Delta t-\lambda^{2}(\Delta t)^{2}+o\left((\Delta t)^{2}\right) \\
\operatorname{Pr}(2 \text { Poisson events in } \Delta t) & =\frac{\lambda^{2}(\Delta t)^{2}}{2} e^{-\lambda \Delta t} \simeq \frac{\lambda^{2}(\Delta t)^{2}}{2}+o\left((\Delta t)^{2}\right) .
\end{aligned}
$$

By ignoring the terms of order greater than $(\Delta t)^{2}$ the probability 3.35 can be rewritten (see [3] 
for details) as follows:

$$
\begin{aligned}
\widehat{p}_{k}(t+2 \Delta t) \simeq & 2 \widehat{p}_{k-1}(t) \frac{\lambda^{2}(\Delta t)^{2}}{2}+\widehat{p}_{k-1}(t) \lambda^{2}(\Delta t)^{2}+ \\
& +\widehat{p}_{k}(t)(1-\lambda \Delta t)^{2}+\widehat{p}_{k}(t) \lambda^{2}(\Delta t)^{2}+ \\
& +2 \operatorname{Pr}(\widehat{N}(t)=k-1, t \in B)\left(\lambda \Delta t-2 \lambda^{2}(\Delta t)^{2}\right)+ \\
& +2 \operatorname{Pr}(\widehat{N}(t)=k, t \in C)\left(\lambda \Delta t-2 \lambda^{2}(\Delta t)^{2}\right)+o\left((\Delta t)^{2}\right) .
\end{aligned}
$$

By a similar reasoning we obtain the following probability, on a single interval of length $\Delta t$

$$
\begin{aligned}
& \widehat{p}_{k}(t+\Delta t) \simeq \widehat{p}_{k-1}(t) \frac{\lambda^{2}(\Delta t)^{2}}{2}+\operatorname{Pr}(\widehat{N}(t)=k-1, t \in B)\left(\lambda \Delta t-\lambda^{2}(\Delta t)^{2}\right) \\
& +\widehat{p}_{k}(t)\left(1-\lambda \Delta t+\frac{\lambda^{2} \Delta t^{2}}{2}\right)+\operatorname{Pr}(\widehat{N}(t)=k, t \in C)\left(\lambda \Delta t-\lambda^{2}(\Delta t)^{2}\right)+o\left((\Delta t)^{2}\right) .
\end{aligned}
$$

We multiply (3.37) by $2(1-\lambda \Delta t)$ and ignore the terms of order greater than $(\Delta t)^{2}$, so that we get

$$
\begin{aligned}
& \widehat{p}_{k-1}(t) \lambda^{2}(\Delta t)^{2} \simeq 2(1-\lambda \Delta t) \widehat{p}_{k}(t+\Delta t)-2 \operatorname{Pr}(\widehat{N}(t)=k-1, t \in B)\left(\lambda \Delta t-2 \lambda^{2}(\Delta t)^{2}\right)+ \\
- & 2 \widehat{p}_{k}(t)\left[(1-\lambda \Delta t)^{2}+\frac{\lambda^{2} \Delta t^{2}}{2}\right]-2 \operatorname{Pr}(\widehat{N}(t)=k, t \in C)\left(\lambda \Delta t-2 \lambda^{2}(\Delta t)^{2}\right)+o\left((\Delta t)^{2}\right),
\end{aligned}
$$

which can be substituted in the first line of (3.36). Finally we obtain

$$
\widehat{p}_{k}(t+2 \Delta t) \simeq \widehat{p}_{k-1}(t) \lambda^{2}(\Delta t)^{2}+2(1-\lambda \Delta t) \widehat{p}_{k}(t+\Delta t)-\widehat{p}_{k}(t)(1-\lambda \Delta t)^{2},
$$

which, divided by $(\Delta t)^{2}$, gives

$$
\frac{\widehat{p}_{k}(t+2 \Delta t)-2 \widehat{p}_{k}(t+\Delta t)+\widehat{p}_{k}(t)}{(\Delta t)^{2}}+2 \lambda \Delta t \frac{\widehat{p}_{k}(t+\Delta t)-\widehat{p}_{k}(t)}{(\Delta t)^{2}}=-\lambda^{2}(\Delta t)^{2} \frac{\widehat{p}_{k}(t)-\widehat{p}_{k-1}(t)}{(\Delta t)^{2}} .
$$

By letting $\Delta t \rightarrow 0$ we easily obtain the second-order equation (3.28).

Remark 3.5 This special case is particularly interesting because it describes a generalization of the Poisson process which have been used in many articles. Random motions at finite velocities spaced by this particular renewal process have been considered by different authors ([6]- [7]-[11] and [23]]).

In particular in [4] we have studied a model with uniformly distributed deviations which take place at even-order Poisson events and therefore its interarrival times are distributed as Gamma( $\lambda, 2)$ (thus the conditional distribution possess a Dirichlet structure).

\section{Conclusions}

The results of the previous sections can be generalized to the $n$-th order case, if we consider the following equation

$$
\frac{d^{n v} p_{k}}{d t^{n v}}+\left(\begin{array}{c}
n \\
1
\end{array}\right) \lambda \frac{d^{(n-1) v} p_{k}}{d t^{(n-1) v}}+\ldots+\left(\begin{array}{c}
n \\
n-1
\end{array}\right) \lambda^{n-1} \frac{d^{v} p_{k}}{d t^{v}}=-\lambda^{n}\left(p_{k}-p_{k-1}\right), \quad k \geq 0,
$$


where $v \in(0,1)$, subject to the initial conditions

$$
\begin{aligned}
p_{k}(0) & =\left\{\begin{array}{ll}
1 & k=0 \\
0 & k \geq 1
\end{array}, \quad \text { for } 0<v<1\right. \\
\left.\frac{d^{j}}{d t^{j}} p_{k}(t)\right|_{t=0} & =0 \quad j=1, \ldots, n-1, \quad k \geq 0, \quad \text { for } \frac{1}{n}<v<1
\end{aligned}
$$

and $p_{-1}(t)=0$. Following the same steps as for the first two models we get the Laplace transform of (4.1):

$$
\left[s^{n v}+\left(\begin{array}{l}
n \\
1
\end{array}\right) \lambda s^{(n-1) v}+\ldots+\left(\begin{array}{c}
n \\
n-1
\end{array}\right) \lambda^{n-1} s^{2 v}+\lambda^{n}\right] \mathscr{L}\left\{\widetilde{p}_{k}^{v}(t) ; s\right\}=\lambda^{n} \mathscr{L}\left\{\widetilde{p}_{k-1}^{v}(t) ; s\right\},
$$

which can be solved recursively (taking into account the initial conditions (4.2)), yielding

$$
\mathscr{L}\left\{\widetilde{p}_{k}^{v}(t) ; s\right\}=\frac{\sum_{j=1}^{n}\left(\begin{array}{c}
n \\
j
\end{array}\right) s^{v j-1} \lambda^{(k+1) n-j}}{\left(s^{v}+\lambda\right)^{(k+1) n}} .
$$

For $n=2$, we obtain from (4.3) formula (3.4). The Laplace transform can be inverted by applying again (2.7) and the solution is given, also in this case, as a sum of GML functions as follows:

$$
\widetilde{p}_{k}^{v}(t)=\sum_{j=1}^{n}\left(\begin{array}{l}
n \\
j
\end{array}\right)\left(\lambda t^{v}\right)^{n(k+1)-j} E_{v, v n(k+1)-v j+1}^{k n+n}\left(-\lambda t^{v}\right) .
$$

For $n=1$, we get the distribution of the first model (2.5), while, for $n=2$, we get the solution of the second-type equation in the form (3.5). In this case the use of (3.6) requires much harder calculations. Nevertheless a relationship similar to (3.8) can be obtained, even for $n>2$, by studying the density $\tilde{f}_{k}^{v}(t), t>0$ of the waiting time of the $k$-th event $T_{k}$. As already seen in section 3 , the following identity must be satisfied by $\widetilde{f}_{k}^{v}(t)$ :

$$
\mathscr{L}\left\{\widetilde{p}_{k}^{v}(t) ; s\right\}=\frac{1}{s} \mathscr{L}\left\{\tilde{f}_{k}^{v}(t) ; s\right\}-\frac{1}{s} \mathscr{L}\left\{\tilde{f}_{k+1}^{v}(t) ; s\right\},
$$

so that, by substituting (4.3) in the l.h.s. of (4.5) we get

$$
\mathscr{L}\left\{\tilde{f}_{k}^{v}(t) ; s\right\}=\frac{\lambda^{n k}}{\left(s^{v}+\lambda\right)^{n k}}
$$

which can be inverted as usual, thus obtaining

$$
\tilde{f}_{k}^{v}(t)=\lambda^{n k} t^{n v k-1} E_{v, n v k}^{n k}\left(-\lambda t^{v}\right)
$$

Again the process is a renewal one, since (4.6) coincides with the sum of $k$ independent and identically distributed random variables $\widetilde{\mathscr{U}_{j}}$ 's (representing the interarrival times) with density given by

$$
\operatorname{Pr}\left\{\widetilde{\mathscr{U}_{j}} \in d t\right\} / d t=\mathscr{L}^{-1}\left\{\frac{\lambda^{n}}{\left(s^{v}+\lambda\right)^{n}} ; t\right\}=\lambda^{n} t^{n v-1} E_{v, n v}^{n}\left(-\lambda t^{v}\right)=f_{1}^{n v}(t) .
$$

Formula (4.7) shows that each interarrival time of the $n$-th order case is distributed as the sum of $n$ independent interarrival times of the first model. This suggests that the following relationship between the corresponding probability distributions holds:

$$
\widetilde{p}_{k}^{v}(t)=p_{n k}^{v}(t)+p_{n k+1}^{v}(t)+\ldots+p_{n k+n-1}^{v}(t), \quad n>2 .
$$




\section{Appendix}

For the reader's convenience we give a short proof of the following Mittag-Leffler integral representation used in (2.22):

$E_{v, \beta}\left(-t^{v}\right)=\frac{t^{1-\beta}}{\pi} \int_{0}^{+\infty} r^{v-\beta} e^{-r t} \frac{\sin (v \pi)}{\left[r^{v}+\cos (v \pi)\right]^{2}+\sin ^{2}(v \pi)}\left[\frac{\sin (\beta \pi)}{\sin (v \pi)}\left[r^{v}-\cos (v \pi)\right]+\cos (\beta \pi)\right] d r$,

for $0<v<1$ and $0<\beta<v+1$. We derive (5.1) by starting from the series expression of the Mittag-Leffler function:

$$
\begin{aligned}
& E_{v, \beta}\left(-t^{v}\right) \\
= & \sum_{m=0}^{\infty} \frac{(-1)^{m} t^{v m}}{\Gamma(v m+\beta)} \frac{\sin ((v m+\beta) \pi)}{\pi} \frac{\pi}{\sin ((v m+\beta) \pi)} \\
= & \frac{1}{\pi} \sum_{m=0}^{\infty} \frac{(-1)^{m} t^{v m}}{\Gamma(v m+\beta)} \frac{\sin ((v m+\beta) \pi)}{\pi} \Gamma(1-v m-\beta) \Gamma(v m+\beta) \\
= & \frac{t^{1-\beta}}{\pi} \sum_{m=0}^{\infty} \frac{(-1)^{m}}{\Gamma(v m+\beta)} \sin ((v m+\beta) \pi) \int_{0}^{+\infty} e^{-r t} r^{-v m-\beta} d r \int_{0}^{+\infty} e^{-y} y^{v m+\beta-1} d y \\
= & \frac{t^{1-\beta}}{\pi} \sum_{m=0}^{\infty} \frac{(-1)^{m}}{\Gamma(v m+\beta)} \sin ((v m+\beta) \pi) \int_{0}^{+\infty} e^{-r t}\left(\int_{0}^{+\infty} e^{-r y} y^{v m+\beta-1} d y\right) d r \\
= & \frac{t^{1-\beta}}{\pi} \int_{0}^{+\infty} e^{-r t}\left(\int_{0}^{+\infty} e^{-r y} y^{\beta-1} \sum_{m=0}^{\infty} \frac{(-1)^{m} y^{v m}}{\Gamma(v m+\beta)} \frac{e^{i \pi v m+i \pi \beta}-e^{-i \pi v m-i \pi \beta}}{2 i} d y\right) d r \\
= & \frac{t^{1-\beta}}{2 \pi i} \int_{0}^{\infty} d y \int_{0}^{\infty} e^{-r(y+t)} y^{\beta-1}\left[e^{i \pi \beta} E_{v, \beta}\left(-y^{v} e^{i \pi v}\right)-e^{-i \pi \beta} E_{v, \beta}\left(-y^{v} e^{-i \pi v}\right)\right] d r \\
= & \frac{t^{1-\beta}}{2 \pi i} \int_{0}^{\infty} e^{-r t}\left[e^{i \pi \beta} \frac{r^{v-\beta}}{r^{v}+e^{i \pi v}}-e^{-i \pi \beta} \frac{r^{v-\beta}}{r^{v}+e^{-i \pi v}}\right] d r \\
= & \frac{t^{1-\beta}}{\pi} \int_{0}^{\infty} e^{-r t} r^{v-\beta} \frac{r^{v} \sin (\pi \beta)+\sin (\pi(\beta-v))}{r^{2 v}+2 r^{v} \cos (\pi v)+1} d r \\
= & \frac{t^{1-\beta}}{\pi} \int_{0}^{\infty} e^{-r t} r^{v-\beta} \frac{\sin (\pi v)}{\left(r^{v}+\cos (\pi v)\right)^{2}+\sin ^{2}(\pi v)} \times \sin (\pi v) \\
& \times\left[r^{v} \frac{\sin (\pi \beta)}{\sin (\pi v)}+\frac{\sin (\pi \beta) \cos (\pi v)-\sin (\pi v) \cos (\pi \beta)}{\Gamma r .}\right] d r
\end{aligned}
$$

From the previous expression, formula (5.1) easily follows and, for $\beta=v$, it reduces to

$$
E_{v, v}\left(-t^{v}\right)=\frac{t^{1-v}}{\pi} \int_{0}^{+\infty} r^{v} e^{-r t} \frac{\sin (v \pi)}{\left[r^{v}+\cos (v \pi)\right]^{2}+\sin ^{2}(v \pi)} d r .
$$


As a check of (5.2) we can study the limit for $v \rightarrow 1:$

$$
\begin{aligned}
E_{v, v}\left(-t^{v}\right)= & \frac{t^{1-v}}{v \pi} \int_{0}^{+\infty} r^{\frac{1}{v}} e^{-r^{\frac{1}{v}}} \frac{\sin (v \pi)}{[r+\cos (v \pi)]^{2}+\sin ^{2}(v \pi)} d r \\
= & \frac{t^{1-v}}{2 \pi v} \int_{0}^{+\infty} r^{\frac{1}{v}} e^{-r^{\frac{1}{v}}}\left(\int_{-\infty}^{+\infty} e^{-i r \beta-|\beta| \sin (\pi v)-i \beta \cos (\pi v)} d \beta\right) d r \\
& \stackrel{v \rightarrow 1}{\rightarrow} \frac{1}{2 \pi} \int_{0}^{+\infty} r e^{-r t}\left(\int_{-\infty}^{+\infty} e^{-i r \beta+i \beta} d \beta\right) d r \\
= & \int_{0}^{+\infty} r e^{-r t} \delta(r-1) d r=e^{-t}=E_{1,1}(-t) .
\end{aligned}
$$

For large $t$, the following approximations follow from (5.1) and (5.2):

$$
E_{v, \beta}\left(-t^{v}\right) \simeq\left\{\begin{array}{l}
\frac{\Gamma(v-\beta+1)}{\pi t^{v}} \sin ((\beta-v) \pi), \quad v \neq \beta \\
\frac{\Gamma(v+1)}{\pi t^{2 v}} \sin (v \pi), \quad v=\beta
\end{array}\right.
$$

In the particular case where $\beta=1$, formula (5.1) reduces to

$$
E_{v, 1}\left(-t^{v}\right)=\frac{\sin (v \pi)}{\pi} \int_{0}^{+\infty} \frac{r^{v-1} e^{-r t}}{r^{2 v}+2 r^{v} \cos (v \pi)+1} d r .
$$

Alternatively, we can rewrite (5.3) in a form which permits us to interpret it as the mean of a Cauchy random variable:

$$
\begin{aligned}
E_{v, 1}\left(-t^{v}\right) & =\frac{1}{\pi} \int_{0}^{+\infty} \frac{\sin (v \pi)}{\left[r^{v}+\cos (v \pi)\right]^{2}+\sin ^{2}(v \pi)} r^{v-1} e^{-r t} d r \\
& =\frac{1}{\pi v} \int_{0}^{+\infty} \frac{\sin (v \pi)}{[r+\cos (v \pi)]^{2}+\sin ^{2}(v \pi)} e^{-r^{1 / v} t} d r \\
& =\mathbb{E}_{X}\left\{\frac{1}{v} e^{-t X^{1 / v}} 1_{[0, \infty)}\right\},
\end{aligned}
$$

where $X$ is distributed as a Cauchy with parameters $-\cos (\pi v)$ and $\sin (v \pi)$. For $v=1$, we can write (5.4), by means of the characteristic function of a Cauchy random variable, as follows:

$$
\begin{aligned}
E_{v, 1}\left(-t^{v}\right)= & \frac{1}{2 \pi v} \int_{0}^{+\infty} e^{-r^{1 / v} t}\left(\int_{-\infty}^{+\infty} e^{-i r \beta-|\beta| \sin (\pi v)-i \beta \cos (\pi v)} d \beta\right) d r \\
& \stackrel{v \rightarrow 1^{-}}{\rightarrow} \int_{0}^{+\infty} e^{-r t} \delta(r-1) d r=e^{-t}=E_{1,1}(-t) .
\end{aligned}
$$


We study now a similar expansion for the Wright function, valid for any $0<v<1$ and $\beta \neq 1$. By following the same steps as in the proof of $(5.1)$, we have that

$$
\begin{aligned}
& W_{v, \beta}\left(-t^{v}\right) \\
& =\sum_{m=0}^{\infty} \frac{(-t)^{v m}}{m ! \Gamma(v m+\beta)} \\
& =\frac{t^{1-\beta}}{2 \pi i} \int_{0}^{\infty} d y \int_{0}^{\infty} e^{-r(y+t)} y^{\beta-1}\left[e^{i \pi \beta} W_{v, \beta}\left(-y^{v} e^{i \pi v}\right)-e^{-i \pi \beta} W_{v, \beta}\left(-y^{v} e^{-i \pi v}\right)\right] d r \\
& =\frac{t^{1-\beta}}{2 \pi i}\left[e^{i \pi \beta} \int_{0}^{\infty} e^{-r t} \sum_{m=0}^{\infty} \frac{\left(-e^{i \pi v}\right)^{m}}{m ! \Gamma(v m+\beta)} \frac{\Gamma(v m+\beta)}{r^{\beta+v m}} d r+\right. \\
& \left.-e^{-i \pi \beta} \int_{0}^{\infty} e^{-r t} \sum_{m=0}^{\infty} \frac{\left(-e^{-i \pi v}\right)^{m}}{m ! \Gamma(v m+\beta)} \frac{\Gamma(v m+\beta)}{r^{\beta+v m}} d r\right] \\
& =\frac{t^{1-\beta}}{2 \pi i} \int_{0}^{\infty} \frac{e^{-r t}}{r^{\beta}}\left[e^{i \pi \beta-\frac{e^{i \pi v}}{r^{v}}}-e^{-i \pi \beta-\frac{e^{-i \pi v}}{r^{v}}}\right] d r \\
& =\frac{t^{1-\beta}}{\pi} \int_{0}^{\infty} \frac{e^{-r t}}{r^{\beta}} e^{-\frac{\cos (\pi v)}{r^{v}}}\left[\frac{e^{i \pi \beta-\frac{i \sin (\pi v) \pi}{\pi r^{v}}}-e^{-i \pi \beta+\frac{i \sin (\pi v) \pi}{\pi r^{v}}}}{2 i}\right] d r \\
& =\frac{t^{1-\beta}}{\pi} \int_{0}^{\infty} \frac{e^{-r t}}{r^{\beta}} e^{-\frac{\cos (\pi v)}{r^{v}}} \sin \left(\pi\left(\beta-\frac{\sin (\pi v)}{\pi r^{v}}\right)\right) d r .
\end{aligned}
$$

\section{References}

[1] Beghin, L. (2008), Pseudoprocesses governed by higher-order fractional differential equations, Electronic Journ. Prob., 13, n.16, 467-485. MR2386739 (2009g:60048)

[2] Beghin, L., Orsingher, E. (2009), Fractional Poisson processes and related planar random motions, Electronic Journ. Prob., 14, n.61, 1790-1826.MR2535014

[3] Beghin, L., Orsingher, E. (2009), Poisson-type processes governed by fractional and higherorder recursive differential equations, arXiv:0910.5855v1, [math.PR], 30 Oct. 2009.

[4] Beghin, L., Orsingher, E. (2010), Moving randomly amid scattered obstacles, Stochastics, vol. 82, n.2, 201-229.

[5] Cahoy, D.O. (2007), Fractional Poisson process in terms of $\alpha$-stable densities, Ph.D. Thesis, Case Western University.

[6] Di Crescenzo, A. (2001), On random motions with velocities alternating at Erlang-distributed random times, Adv. in Appl. Probab. 33 (3), 690-701.MR1860096 (2002h:60231)

[7] Di Crescenzo, A. (2002), Exact transient analysis of a planar random motion with three directions, Stoch. Stoch. Rep., 72, n. 3-4, 175-189. MR1897914 (2003c:60091) 
[8] Gorenflo R., Mainardi F. (1997), Fractional calculus, integral and differential equations of fractional order, in Fractals and Fractional Calculus in Continuum Mechanics, A.Carpinteri and F.Mainardi Editors, Springer Verlag, Wien, n.378, Series CISM Courses and Lecture Notes, 223-276. MR1611585 (99g:26015)

[9] Gradshteyn, I. S., Ryzhik, I. M. (2000), Table of integrals, series, and products. Translated from the Russian. Sixth edition. Translation edited and with a preface by Alan Jeffrey and Daniel Zwillinger. Academic Press, Inc., San Diego, CA. MR1773820 (2001c:00002)

[10] Jumarie, G. (2001), Fractional master equation: non-standard analysis and LiouvilleRiemann derivative, Chaos, Solitons and Fractals, 12, 2577-2587. MR1851079 (2003i:82069)

[11] Lachal, A. (2006), Cyclic random motions in $\mathbb{R}^{d}$-space with $n$ direction,. ESAIM Probab. Stat, . 10, 277-316. MR2247923 (2007k:60036)

[12] Lageras, A. N (2005), A renewal-process-type expression for the moments of inverse subordinators, Journ. of Applied Prob., 42, 1134-1144. MR2203828 (2007c:60089)

[13] Laskin, N. (2003), Fractional Poisson process, Communications in Nonlinear Science and Numerical Simulation, 8, 201-213. MR2007003 (2004j:60101)

[14] Mainardi F. (1996), The fundamental solutions for the fractional diffusion-wave equation, Applied Mathematics Letters, 9, n.6, 23-28. MR1419811 (97h:35132)

[15] Mainardi F. (1996), Fractional relaxation-oscillation and fractional diffusion-wave phenomena, Chaos, Solitons and Fractals, 7, n.9, 1461-1477. MR1409912 (97i:26011)

[16] Mainardi F., Raberto, M., Gorenflo R., Scalas E. (2000), Fractional calculus and continuoustime finance II: the waiting-time distribution, Physica A, 287, 468-481.

[17] Mainardi F., Gorenflo R., Scalas E. (2004), A fractional generalization of the Poisson processes, Vietnam Journ. Math., 32, 53-64. MR2120631

[18] Mainardi F., Gorenflo R., Vivoli A. (2005), Renewal processes of Mittag-Leffler and Wright type, Fractional Calculus and Applied Analysis, 8, (1), 7-38. MR2179226

[19] Mainardi, F, Gorenflo R., Vivoli A. (2007), Beyond the Poisson renewal process: A tutorial survey, Journ. of Computational and Applied Mathematics, 205, 725-735. MR2329648

[20] Orsingher, E., Beghin, L. (2004), Time-fractional equations and telegraph processes with Brownian time, Probability Theory and Related Fields, 128, 141-160. MR2027298 (2005a:60056)

[21] Orsingher, E., Beghin, L. (2009), Fractional diffusion equations and processes with randomly-varying time, Annals of Probability, 37 (1), 206-249. MR2489164 (2010b:60116)

[22] Podlubny, I. (1999), Fractional Differential Equations, Academic Press, San Diego. MR1658022 (99m:26009)

[23] Pogorui, A.A., Rodrìguez-Dagnino, R.M. (2005), One-dimensional semi-Markov evolutions with general Erlang sojourn times, Random Oper. Stoch. Equat., 13, (4), 399-405. MR2183564 (2007g:60107) 
[24] Prabhakar T.R. (1971), A singular integral equation with a generalized Mittag Leffler function in the kernel, Yokohama Math. J. 19, 7-15. MR0293349

[25] Repin O.N., Saichev, A.I. (2000), Fractional Poisson law, Radiophysics and Quantum Electronics, 43 (9), 738-741. MR1910034

[26] Saji Kumar V.R., Pillai, R.N. (2006), Single server queue with batch arrivals and $\alpha$-Poisson distribution, Calcutta Stat. Assoc. Bull., 58, n.229-230, 93-103. MR2305408 (2007m:60282)

[27] Saxena, R.K., Mathai, A.M., Haubold, H.J. (2006), Fractional reaction-diffusion equations, Astrophysics and Space Science, 305, 289-296.

[28] Saxena, R.K., Mathai, A.M., Haubold, H.J., (2006), Solutions of fractional reaction-diffusion equations in terms of Mittag-Leffler functions, Intern. Journ. Scient. Research, 15, 1-17. arXiv:0708.2265 (August 2007).

[29] Shukla A.K., Prajapati, J.C., (2007), On a generalization of Mittag-Leffler function and their properties, Journ. Math. Anal. Appl., 336, 797-811. 33E12 (33C15 33E20 44A20)

[30] Uchaikin, V.V., Sibatov, R.T. (2008), Fractional theory for transport in disordered semiconductors, Communic. Nonlinear Sciences and Numerical Simulation, 13, 715-727. MR2381497 (2008k:82136) 